\title{
Validation of Habitat Assessment Services in Natura 2000 Sites: Streamlining the Role of Stakeholders from User Needs to Conservation Status Evaluation
}

Christoph SCHRÖDER ${ }^{1}$, Francisco Javier BONET GARCIA ${ }^{2}$, Oliver BUCK $^{3}$, Christina CORBANE ${ }^{4}$, Michel DESHAYES ${ }^{4}$, Michael FÖRSTER ${ }^{5}$, Virginia GARCÍA MILLÁN ${ }^{3}$, Yolanda GIL JIMENEZ ${ }^{6}$, Stefan LANG $^{7}$, Blanca RAMOS LOSADA ${ }^{8}$, Alejandro SIMÓN COLINA ${ }^{9}$ and Jeroen VANDEN BORRE ${ }^{10}$

${ }^{1}$ Universidad de Málaga/Spain · christoph.schroder@uma.es ${ }^{2}$ Universidad de Granada/Spain ${ }^{3}$ EFTAS GmbH, Münster/Germany ${ }^{4}$ IRSTEA, Montpelier/France

${ }^{5}$ TU Berlin/Germany ${ }^{6}$ Agencia de Medio Ambiente y Agua de Andalucía, Sevilla/Spain

${ }^{7}$ Department of Geoinformatics - Z_GIS, University of Salzburg/Austria

${ }^{8}$ Parque Nacional Sierra Nevada, Granada/Spain ${ }^{9}$ EU SatCen, Madrid/Spain

${ }^{10} \mathrm{INBO}$, Brussels/Belgium

This contribution was double-blind reviewed as extended abstract.

\begin{abstract}
A key success factor of the Copernicus programme is to ensure the acceptance of services by users. This acceptance and further adoption is based on high quality products that meet the specific information requirements of the user. In the realm of biodiversity monitoring and reporting on conservation status of Natura 2000 habitats, such products must meet different needs of local site manager and national environmental agencies, up to European authorities. Hence, the quality of these upcoming Earth observation based technologies must be validated in a pre-operational phase. The case of the MS.MONINA project shows the necessity to streamline the role of different stakeholders in the whole service delivery process. This process is described in depth, taken from the service validation in Sierra Nevada and Andalusia (Spain), showing potentials and limitations of the developed validation protocol and highlighting methodological, technical and scientific achievements obtained during this process.
\end{abstract}

\section{Introduction}

The FP7 project MS.MONINA (Multi-scale Service for Monitoring Natura 2000 Habitats of European Community Interest, grant agreement No. 263479) ${ }^{1}$ offers Earth observation (EO)-based monitoring services for detecting and managing the state of Natura 2000 sites and other precious habitats to reduce the loss of biodiversity. New concepts and methods combining EO-data and in-situ data are defined and implemented with the aim to support

1 www.ms-monina.eu

Jekel, T., Car, A., Strobl, J. \& Griesebner, G. (Eds.) (2013): GI_Forum 2013. Creating the GISociety. (C) Herbert Wichmann Verlag, VDE VERLAG GMBH, Berlin/Offenbach. ISBN 978-3-87907-532-4. C ÖAW Verlag, Wien. eISBN 978-3-7001-7438-7, doi:10.1553/giscience2013s548. 
public authorities at all geographical levels in implementing policies and measures. The intended monitoring capacity is not only at the benefit of national authorities, but also addressing local authorities that manage these sites, and EU authorities overseeing the overall development of the Natura 2000 programme. Based on the major advances that have been achieved in EO based habitat monitoring (e.g. VANDEN BORRE et al. 2011, SPANHOVE et al. 2012), MS.MONINA partner organisations have developed cutting-edge technologies for habitat distribution mapping and conservation status indicators for very different pilot sites, located in four different biogeographical regions. As a result, the services provide tools to bridge the generic statements of the habitat directive and its local implementation in a site. This paper aims at showing (a) the role of users and service providers as stakeholders in the development and assessment of EO based monitoring services for Natura 2000 habitats and (b) validation as a fundamental part of this service development. The five key issues of service validation discussed by ZEIL \& LANG (2009) (see Fig. 1) provide a framework for the stages of the service delivery process to be validated.

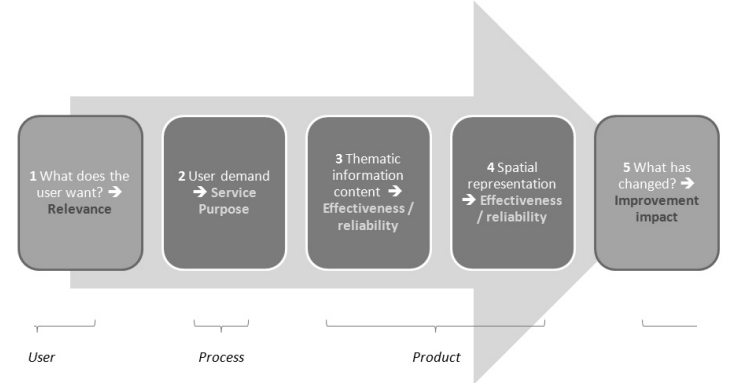

Fig. 1:

Service/product delivery process user domain (red), service provider domain (blue) (ZEIL \& LANG 2009)

In order to showcase the overall service delivery process and assess the services developed during the project's lifetime, a validation exercise was set up for site and state level services. The services responded to user requirements from two independent users in Andalusia (Spain), i.e. Sierra Nevada National and Nature Parks and the Andalusian Government Agency for Environment and Water Agency.

\section{User Requirements and Service Development}

Users are key stakeholder in the development of any product or service. Their requirements regarding the product or service should be the guideline for service provider. Apart from technical and scientific soundness, the overall aim of the service outcomes should be to meet the user requirements satisfactorily. Thus, a fluent communication between users and service providers is crucial for fulfilling this goal. This is even more important in the framework of the Copernicus programme where the user engagement and user perspective is crucial to efficient service development and sustainable service uptake. These premises have been followed by the MS.MONINA project. As part of the validation process, local and regional users and service provider have reached a common understanding of the user requirements and demands, on the one hand, and the technological feasibility on the other hand. A user request form was elaborated and completed by the user, Service provider and users set up the final decision of the service to develop. Several obstacles (e.g. language, 
technical understanding, scientific vs. administrative interest) had to be overcome before defining a concrete and feasible service. The following services were agreed upon by users and service providers for the site level: (1) Forest and bush encroachment and changes/dynamics in Quercus pyrenaica forests, (2) Bush encroachment and changes/dynamics in Juniperus communis formations, (3) Evaluation of restoration activities in $Q$. pyrenaica potential areas, (4) Evaluating pressures of skiing resorts over habitats. The services already developed during the project's lifetime had to be adapted to the local and regional specificities as well as to the available EO and reference data. A multi-temporal set of veryhigh resolution EO data sets were identified to be suitable and specifically acquired and pre-processed for the validation process: WorldView-II, 8 bands, August 2011, QuickBird, 4 bands, August 2008, IKONOS, 4 bands, July 2003. The services could be well adapted to the new settings and conditions. As main projects, habitat distribution and density maps, including change maps as well as indicator maps for conservation status have been produced and delivered for validation to the local and regional users.

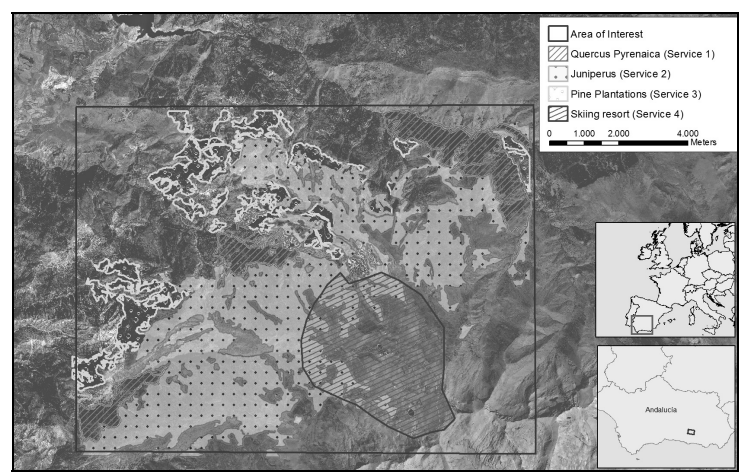

Fig. 2:

Overview map of validation site Sierra Nevada, showing the service related thematic layer

\section{Service Validation}

Validation starts and ends from the user perspective. It can be defined as "a producerindependent process generating documented evidence to which degree the object subject to validation reaches predetermined requirements" (BROGLIA et al. 2010) and aims at helping ascertain the sustainability of service provision. Validation requires a structured communication between user and provider and, hence, is performed at the interface between end user and provider (ZEIL \& LANG 2009). A protocol for the service validation was elaborated following the general framework of the protocol designed in the GMES project SAFER (BROGLIA et al. 2010). The protocol includes four validation categories looking at the reliability of the information content and the consistency of the information support, as well as on the usability of the product and the efficiency of the service. The first two are more focusing on technical parameters, while the latter are more oriented towards a valuation of the service. The Sierra Nevada Observatory of Global Change as well as the regional REDIAM network for environmental information, created and maintained by the Regional Environmental body, also responsible for both user organisations (Sierra Nevada National and Nature Park and the Environment and Water Agency) offer a wide range of already existing information the services could be validated against. As the validation is still on- 
going, final results will only be available for the celebration of the special workshop at the G_Forum 2013.

\section{Results}

As a primary result, an adapted validation protocol for EO based biodiversity monitoring products was developed. The potentials and limitations of these have been shown during the validation phase. Additionally, the validation process has shown the importance of a continuous, structured and collaborative communication between the involved stakeholders as well as the existing obstacles of communication in an international setting. Finally, the results of the service developments are still pending, but promise scientifically and technologically sound developments that support local and regional users in their reporting and monitoring obligations derived from the Habitat Directive.

\section{Conclusion and Outlook}

The monitoring of biodiversity is depending on various aspects, such as habitat type, definition of conservation status and potential disturbances. Therefore, a tailored solution per region has to be found on site and state level. Nevertheless, it is possible to validate such services. Although the service developments and validation are still pending, the generated network of stakeholders has already originated a technology and knowledge transfer between stakeholders and regions that has the potential to boost biodiversity monitoring in the future. This will help to increase the awareness, acceptance and adoption of EO based biodiversity monitoring services by the addressed user groups on local, national and European scale.

\section{References}

Broglia, M., Corbane, C., Carrion, D., Lemoine, G. \& Pesaresi, M. (2010), Validation Protocol for Emergency Response Geo-information Products. JRC Scientific and Technical Reports. Luxembourg: Publications Office of the European Union.

Spanhove, T., Vanden Borre, J., Delalieux, S., Haest, B. \& PaelinckX, D. (2012), Can remote sensing estimate fine-scale quality indicators of natural habitats? Ecological Indicators 18, 403-412.

Vanden Borre, J., Paelinckx, D., Mücher, C.A., Kooistra, L., Haest, B., De Blust, G. \& SCHMIDT, A. M. (2011), Integrating remote sensing in Natura 2000 habitat monitoring: Prospects on the way forward. Journal for Nature Conservation, 19 (2), 116-125.

ZEIL, P. \& LANG, S. (2009), Do have clients a role in validation? In: Corbane, C., Broglia, M., Carrion, D., Lemoine, G. \& Pesaresi, M. (Eds.), VAlgEO - International workshop on validation of geo-information products for crisis management. Ispra, Italy, European Communities, 143-147. 